\title{
Was war los im Palmengarten 2009?
}

\author{
Andrea Mueller \& Matthias Jenny
}

\section{Januar}

Im Palmengarten macht der extrem kalte Winter Pause. Denn mit einem Ausflug in die Tropen kann man dem kalten Frankfurter Winterwetter entfliehen: von minus $14^{\circ} \mathrm{C}$ auf plus $24^{\circ} \mathrm{C}$ in zwei Minuten, das geht im Palmengarten.

In der Kamelien-Ausstellung überwiegt bei der Eröffnung die Farbe Grün, aber nach und nach öffnen die Kamelien ihre Blütenknospen. Neben den Pflanzen werden Bilder von RenATe TessMAR-LEONARDY gezeigt. Die Künstlerin stellt ihre Werke unter dem Titel „Kamelien treffen Kamele" aus und bringt, inspiriert von der Namensähnlichkeit der pflanzlichen und tierischen Kreaturen, Wüste und gemäßigt feuchte Klimate zusammen. Die Kamelienschau ist die vorerst letzte Ausstellung in den Galerien am Palmenhaus, bevor diese für fast ein Jahr wegen Renovierungsarbeiten nicht mehr zugänglich sein werden.

Groß abgedruckt werden in einer Zeitung Fotos aus dem Palmengarten im Jahr 1915. Die Bilder zeigen ein junges Mädchen samt Großund Urgroßmutter beim sonntäglichen Ausflug im Palmengarten. Zur Verfügung gestellt hat sie die mittlerweile 70-jährige Tochter des Mädchens auf dem Foto. Die Nachfahrin erinnert daran, dass es für ihre Mutter und ganze Generationen das Größte war, den Palmengarten zu besuchen. „Früher ist man ja nicht in Urlaub gefahren. Da ist man in den Palmengarten gegangen! Das war Erholung und Unterhaltung in einem. Es gab Konzerte und Tanzveranstaltungen".

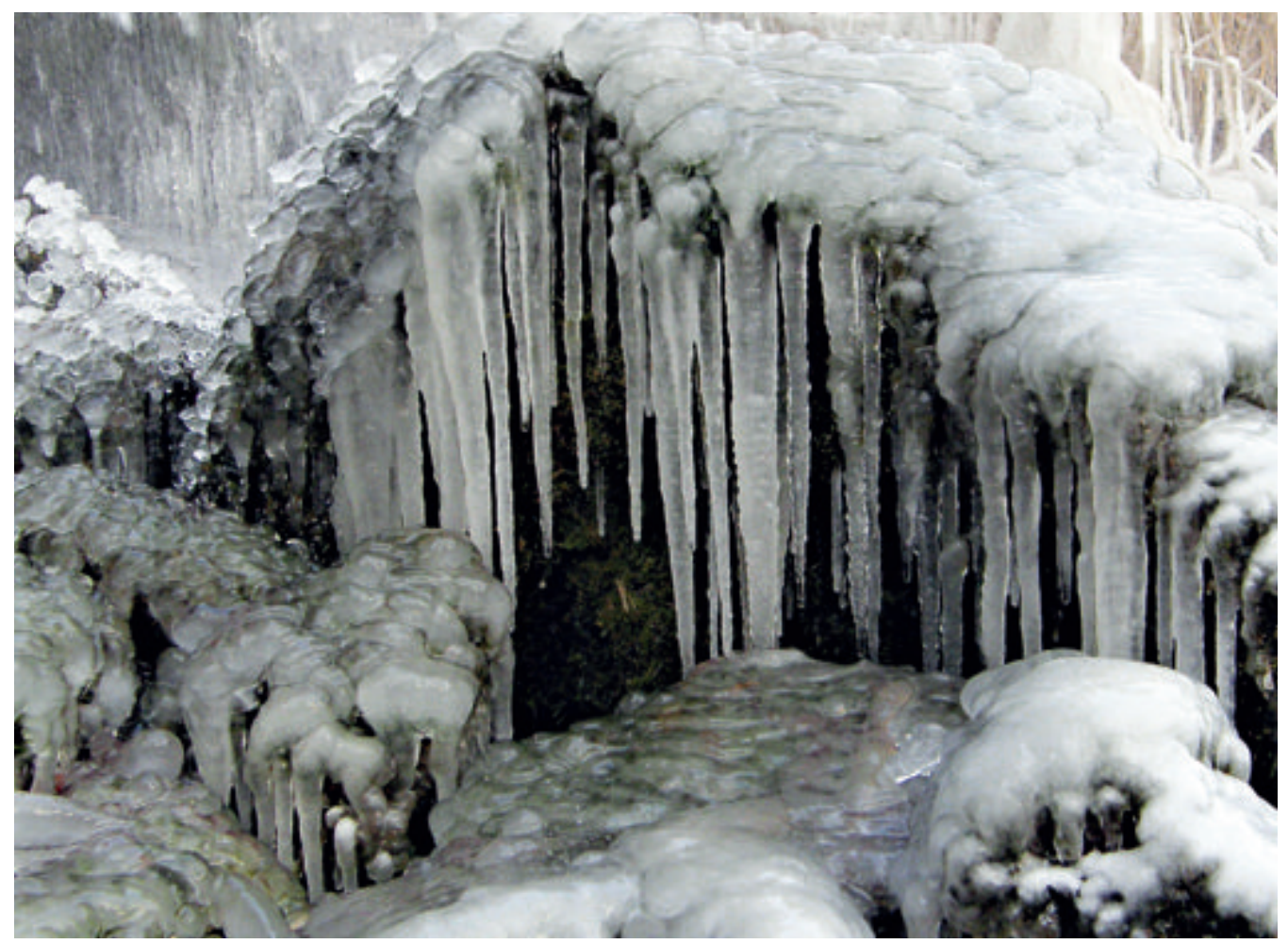

Abb. 1: Vereister Wasserfall als Auftakt für einen extrem harten Winter. 
Tiere im Palmengarten leiden unter der Kälte: Ein Eisvogel zeigt sich regelmäßig im Palmengarten. Der Vogel des Jahres 2009 präsentiert sich mit seinem schönen Federschmuck in der Nähe des Tropicariums. Im geheizten Teich findet er Nahrung. Helmut STEINBACH, von 1955 bis 1994 Leiter der Palmengartenkonzerte, wird 80 Jahre alt. Am ersten Weihnachtsfeiertag 1955 hob der Dirigent zum ersten Mal den Taktstock in seinem neuen Amt als universal verantwortlicher Leiter der Frankfurter Palmengarten-Konzerte. Fortan bescherte STEINBACH seinem Publikum jahrzehntelang Konzerterlebnisse und machte das Gesellschaftshaus des Palmengartens zu einem kulturellen Treffpunkt.

\section{Februar}

Das Jahr 2009 ist dem Naturforscher CHARLES DARWIN gewidmet, dessen 200. Geburtstag in diesem Jahr ebenso gefeiert wird wie der 150 . Jahrestag der Veröffentlichung seines wichtigsten Werkes „Über die Entstehung der Arten“. Hierzu gibt es ein umfangreiches Programm: Vorträge,

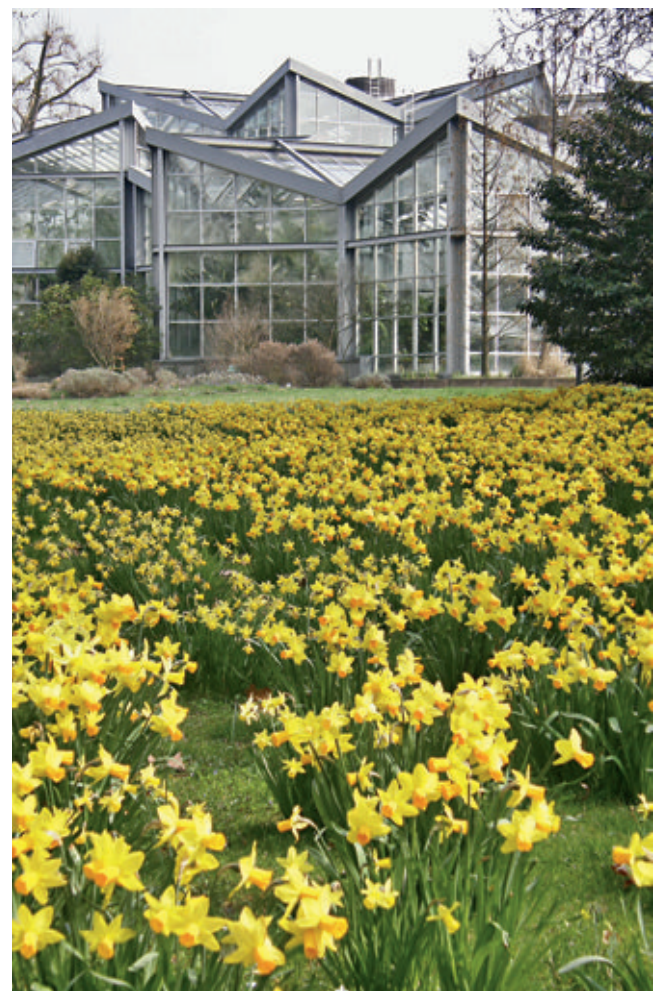

Podiumsdiskussionen, Führungen, Ausstellungen und Veranstaltungen, die von BioFrankfurt organisiert werden. Auch im Palmengarten finden Veranstaltungen dazu statt, wie der Vortrag "Charles DarWin und die Pflanzen“ von STEFAn SCHneckenburger, ehemals Kustos im Palmengarten, jetzt Leiter des Botanischen Gartens Darmstadt.

Zumindest in der Frühlingsblumen-Ausstellung ist ein baldiges Ende des Winters in Sicht. Tausende Tulpen, Krokusse und Narzissen bescheren den Besuchern erste Frühlingsgefühle. Die Ausstellung wird bei noch sehr unfrühlingshaften $4{ }^{\circ} \mathrm{C}$ in einem Zelt über dem Oktogonbrunnen eröffnet. Der Umzug ist den schon lang geplanten und notwendigen Renovierungsarbeiten in der Galerie geschuldet. Neben den Frühlingsblumen wird die Bildersammlung „Temple of Flora" des Engländers ROBERT JOHN THORNTON gezeigt, der im frühen 19. Jh. eine großartige Bildersammlung angelegt hat. THORNTON (1768-1837) war Mediziner, doch seine Leidenschaft galt der Botanik. Er engagierte die bekanntesten Blumenmaler seiner Zeit, um dieses Werk zu schaffen. Im Palmengarten sind Nachdrucke seiner Zeichnungen zu sehen.

Die Grüne Schule bietet während der Frühlingsblumen-Ausstellung Nachwuchs-Botanikern die Gelegenheit, den Geheimnissen der Frühblüher auf die Spur zu kommen.

Grünes Licht für „Kinder im Garten“: Das bundesweit einzigartige Projekt „Kinder im Garten“, ein Gemeinschaftsprojekt der Städtischen Kitas und des Palmengartens, wurde im Palmengarten speziell für Kinder zwischen 3 und 6 Jahren entwickelt. Ab Juni sollen zunächst Kinder aus 12 Kitas pflanzen, experimentieren, kochen und mikroskopieren. Rund 5000 Kinder werden jährlich in der Forschungsstation im Haus Leonhardsbrunn und auf Forschungsreisen durch den Palmengarten erste naturwissenschaftliche Experimente machen und in einer Kinderküche Erfahrungen mit Früchten, Kräutern und Gewürzen sammeln können. Begleitet werden die kleinen Forscher nicht nur von Pädagoginnen

Abb. 2: Üppig blühende Narzissenwiese mit Blick auf das Tropicarium. 
und Erzieherinnen, sondern auch von einer Gärtnerin und einer promovierten Botanikerin. Das erste Thema lautet Bambus. Das Projekt wird so positiv bewertet, dass es für drei Jahre von Stiftungen finanziert wird und nachher von der Stadt weiter gefördert werden soll.

Das Palmengarten-Gesellschaftshaus, das seit 2002 wegen Sicherheitsmängeln nicht mehr genutzt werden kann, wird nun endlich restauriert. Die Verhandlungen mit Bauunternehmern haben sich nach europaweiter Ausschreibung lange hingezogen und als schwierig erwiesen. Nun wurde, nach monatelanger Verzögerung, ein Generalunternehmer gefunden, der die Sanierung übernehmen kann. Die Stilgeschichte des Gesellschaftshauses zeigt, dass es nicht einfach wird. Denn der Festsaal stammt aus derselben Epoche wie die Alte Oper, der südliche Teil im Bauhausstil trägt die Handschrift von MARTIN ELSÄSSER und der Westteil zeugt vom Umbau in den 50er-Jahren. Voraussichtlich wird das Gesellschaftshaus im Frühjahr 2011 wieder in neuem Glanz eröffnet werden. Umweltdezernentin Dr. MANUELA ROTTMANN hat sich nach dem langen Warten noch nie so auf einen Bauzaun gefreut wie auf diesen. Anfang April soll es losgehen.

\section{März}

Die Verkaufsausstellung Garten 2009 findet erstmals erst in der zweiten Märzhälfte statt, zu oft hat es auf die Frühlingshoffnungen geschneit. Der strahlende Sonnenschein bei der Eröffnung am 19. März gibt uns recht. Wegen der beginnenden Sanierungsarbeiten am Gesellschaftshaus konzentriert sich die Gartenmesse auf die nördliche Hälfte des Palmengartens. Ein abwechslungsreiches Rahmenprogramm steigert die Attraktivität der Ausstellung. So stellt unter anderem Eva DEMSKI ihr jüngstes Werk „Gartengeschichten " im Siesmayersaal vor.

An einem Freitag, dem 13. März, werden vier Pinien im Palmengarten gefällt. Zum Bedauern vieler Mitarbeiter und Besucher müssen sie den Bauarbeiten weichen. Es sprechen denkmalpflegerische und gartengestalterische Gründe für die Fällung der Pinien. Eine Verpflanzung kommt wegen ihrer Größe nicht in Frage.
Araber, hat die Tourismus und Congress GmbH Frankfurt (TCF) festgestellt, haben Frankfurt als attraktives Städtereiseziel entdeckt. Nach München ist Frankfurt die beliebteste deutsche Stadt bei arabischen Touristen. Sie mögen vor allem attraktive Landschaften und Orte, der Palmengarten spielt eine bedeutende Rolle.

\section{April}

Fast zwei Wochen nach seinem kalendarischen Anfang hält der Frühling nun endlich Einzug in Frankfurt. Anfang April herrscht die erste stabile Schönwetterlage seit Mitte Januar. Für die Besucher gibt es außer den Frühjahrsblühern die ganze Pracht der Orchideen zu bewundern. Zusammen mit dem Verband Deutscher Orchideenbetriebe zeigt die Orchideen-Ausstellung die Königin der Blumen in allen Farben und Formen.

Der japanische Generalkonsul YosHITAKA HANADA tauft mit großem Trommelwirbel zur Eröffnung der Azaleen-Ausstellung eine neue Sorte auf den Namen „Kinku Saku“. Die neue Züchtung soll besonders winterhart sein, ihre weißrosa Blütenblätter sind lang und schmal. Zur Taufe schlägt ein Trommelkünstler eine japanische Odaiko. Außerdem ist in der Azaleen-Schau eine Kunstausstellung des schwedischen Fotografen EDVARD KoINBERG zu bewundern. Fasziniert von CARL VON LINNÉs Schriften zeigt er die Pflanzen von ihrer erotischen Seite. „Herbarium Amoris“ ist ein modernes calendarium florae, ein Blühkalender, der das Jahr über den Lebenszyklus der Pflanzen beschreibt.

Das Jahresprogramm 2009 von GartenRheinMain, mit insgesamt rund 300 Veranstaltungen, wird im Palmengarten vorgestellt. Das Motto der regionalen Veranstaltungsreihe heißt dieses Jahr „GartenRheinMain bittet zu Tisch“. Dazu wird jeden Monat Nahrhaftes und Leckeres aus Gärten und Parks präsentiert. Der Palmengarten wird im November in dieser Reihe „Schokolade und ihre würzigen Begleiter" anbieten.

$\mathrm{Zu}$ Ostern stehen die Magnolien schon in voller Blüte und die Narzissenwiesen leuchten gelb. Auch der Taschentuchbaum blüht schon. Pfingstrosen sind statt zu Pfingsten in diesem 

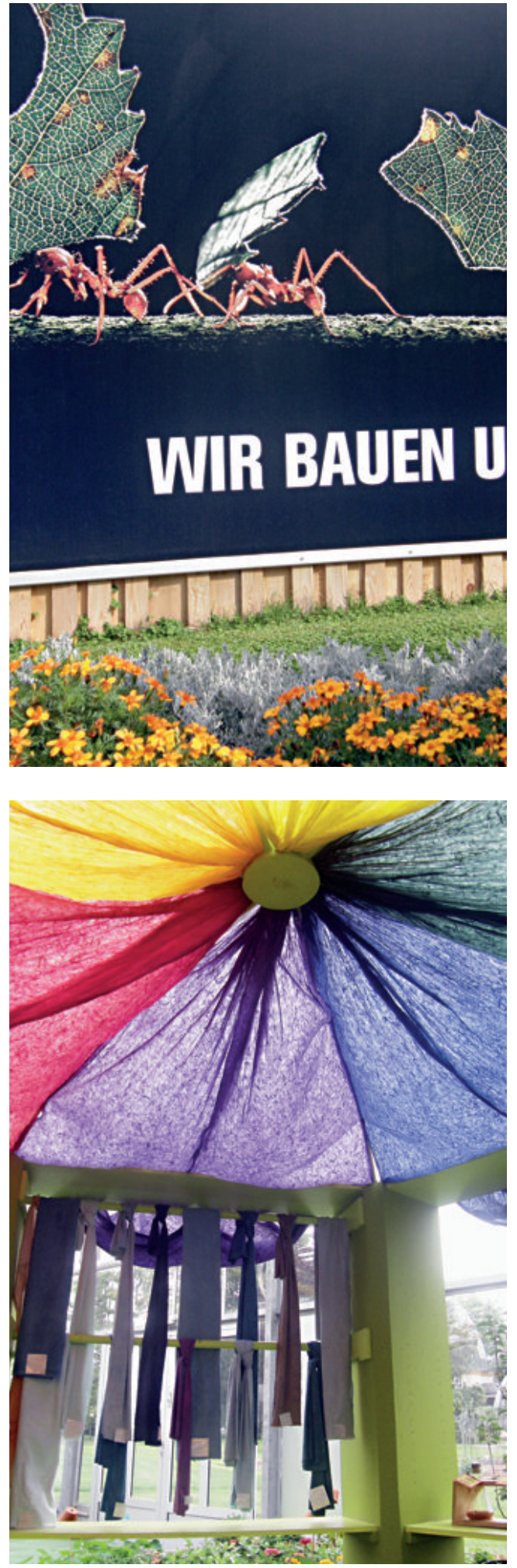

Jahr schon zu Ostern zu bewundern. Das diesjährige Osterfest ist mit Temperaturen um $25^{\circ} \mathrm{C}$ das wärmste der vergangenen hundert Jahre. Tausende sind gekommen, um bei diesem Wetter Ostern unter Palmen zu feiern.

Auf dem wahrscheinlich schönsten Bauzaun Frankfurts arbeiten Blattschneiderameisen. Das Motiv wurde im Frankfurter Zoo fotografiert und ziert nun den Bauzaun am Gesellschaftshaus. Die Arbeiten sollen im Frühjahr 2011 abgeschlossen sein.

\section{Mai}

Am ersten Mai-Wochenende kann man im Palmengarten schon den blühenden Rhododendrongarten bewundern. Trotz des harten Winters gab es kaum Ausfälle bei unseren Pflanzen. Die Dramatische Bühne Frankfurt entführt die Besucher im Palmengarten auf eine Fantasiereise. Mit dem Stück „ALICE im Wunderland“ begeben sich Schauspieler und Zuschauer auf eine Entdeckungstour durch den Garten und erleben dabei viele merkwürdige Überraschungen.

Am 14. Mai wird die große Informationsausstellung „Farbe in der Natur“ im Gewächshaus auf der Spielwiese eröffnet. Auch das Freiland ist vom Thema Farbe inspiriert. Die Beete sind dieses Jahr nach diesem Motto angelegt und bunte Fahnen weisen den Weg zur Ausstellung.

\section{Juni}

Für die Rosenschau hat das Galerieteam sieben verschiedenen Gärten rund um den Oktogonbrunnen angelegt. Im Bauerngarten wachsen Stockrosen gemeinsam mit Rhabarber, Mais und Mangold. Im griechischen Garten ragt ein steinerner ADONIS zwischen marmornen Säulen und weißen Rosen hervor. Der orientalische Garten überrascht mit farbigen Mosaikfliesen. Aufgrund der noch andauernden Renovierungsarbeiten in den Galerien findet die Ausstellung im Freien unter Pavillons statt. Nicht zuletzt wegen des

Abb. 3 (oben): Fleißige Ameisen auf dem Bauzaun am Gesellschaftshaus.

Abb. 4 (unten): Pavillon mit gefärbten Stoffen in der Ausstellung „Farbe in der Natur“. 
guten Wetters ist dies ein voller Erfolg und wunderschön, viele Besucher wünschen sich die Rosenausstellung immer im Freien, doch wir wollen den Wettergott nicht alljährlich herausfordern.

Zum Familienfest lädt die Gemeinnützige Wohnungsbaugesellschaft (GWH) in den Palmengarten. Das bunte Kinderprogramm orientiert sich ganz am aktuellen Ausstellungs-Motto „Farbe in der Natur".

Insektivorenbörse und Jahreshauptversammlung der Gesellschaft der Fleischfressenden Pflanzen finden dieses Jahr im Palmengarten statt.

\section{Juli}

"Jazz im Palmengarten“ ist die älteste kontinuierliche Open-Air-Jazz-Veranstaltung der Welt. Nicht zuletzt hat die einmalige Atmosphäre dazu beigetragen, dass Spitzenmusiker aus aller Welt hier seit fünfzig Jahren mit großer Begeisterung auftreten. Im Jahre 1959 empfiehlt der junge Posaunist AlberT Mangelsdorff dem Palmengartendirektor FRITZ ENCKE, mit Jazz-Konzerten ein jüngeres Publikum in Frankfurts schönsten Garten zu holen. Albert Mangelsdorff gab am 3. Juli 1959 das allererste Konzert. 50 Jahre später gibt es „Jazz im Palmengarten“ immer noch. Kulturdezernent Felix SEMmelroth eröffnet die Jubiläums-Saison. Das Eröffnungskonzert bestreiten die Frankfurt Jazz-Big-Band mit Wilson De Oliveira und Stargast EnRIQue TELLERIA.

Die Kammeroper Frankfurt hat sich mit VERDIs „Rigoletto“ ein gewichtiges Werk vorgenommen. Die Sommerblumen stehen in dieser Jahreszeit in duftender Blütenpracht. Aus Anlass ihres 90sten Geburtstages wird das Beet am Tropicarium der Naturschützerin HANNELORE SCHMIDT gewidmet. In 90 Städten haben die Stiftung LOKI SCHMIDT und die Stiftung Naturschutz in Hamburg bundesweit Parks und Gärten gewonnen, für jedes Lebensjahr von LOKI SCHMIDT ein Beet anzulegen. „Das ist das schönste Geburtstagsgeschenk, das ich je erhalten habe“, sagt die Jubilarin bei ihrem Wiegenfest. Im Sommerflor wimmelt es nur so von Distel-

Abb. 5: LOKI SCHMIDT-Beet im Palmengarten.

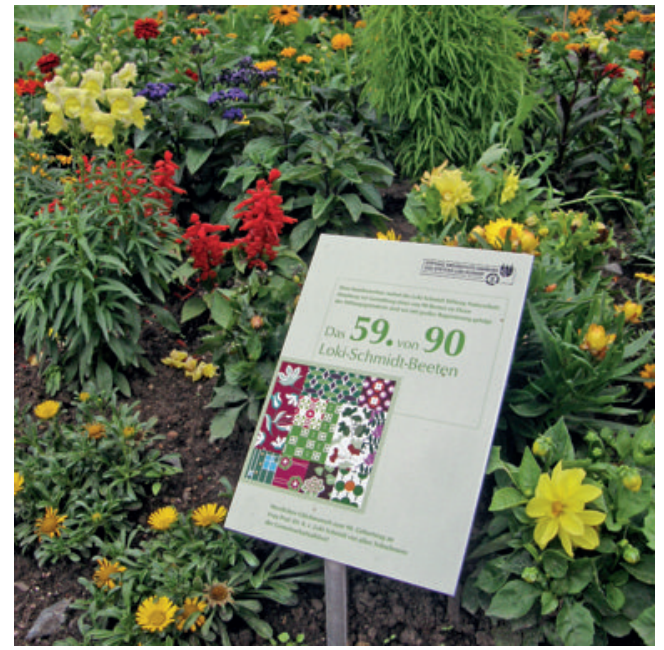

faltern. Bundesweit wird von einer Falter-Invasion gesprochen.

Pünktlich zum letzten Juli-Wochenende und damit äußerst besucherfreundlich blüht die Titanenwurz im Mangroven-Haus. Die stinkende Pracht misst diesmal $1,81 \mathrm{~m}$ und welkt bereits nach zwei Tagen.

\section{August}

Vier Monate nach dem Beginn der Bauarbeiten am Gesellschaftshaus klafft ein großes Loch im historischen Festsaal. Der Gewölbekeller darunter wurde freigelegt, damit dort später ein absenkbares Podium zur Vergrößerung der Bühne eingebaut werden kann. Mittlerweile sind im ganzen Saal die Verschalungen und Einbauten der 50er Jahre abgerissen worden. Der Blick ist frei auf den Saal mit Stuck und Gemälden, wie er Ende des 19. Jh. ausgesehen hat. Zur Freude der Architekten sind diese sogar besser erhalten als erwartet.

Im Palmengarten wird am 20. August geerntet und musiziert. HARRIET LEWIS aus Philadelphia zeigt, wie rau, gefährlich und traurig ihre Musik sein kann. LEWIS, die u.a. mit MICHAEL JACKSON als Backgroundsängerin auf Tournee war, tritt bei „Blues im Palmengarten“ auf. Am gleichen Tag werden im Tropicarium die Bananen geerntet. Eine Staude liefert über 70 Bananen.

Mit „Kennen Sie PuCCInI?" eröffnet das Papageno Musiktheater die neue Spielzeit. Die 

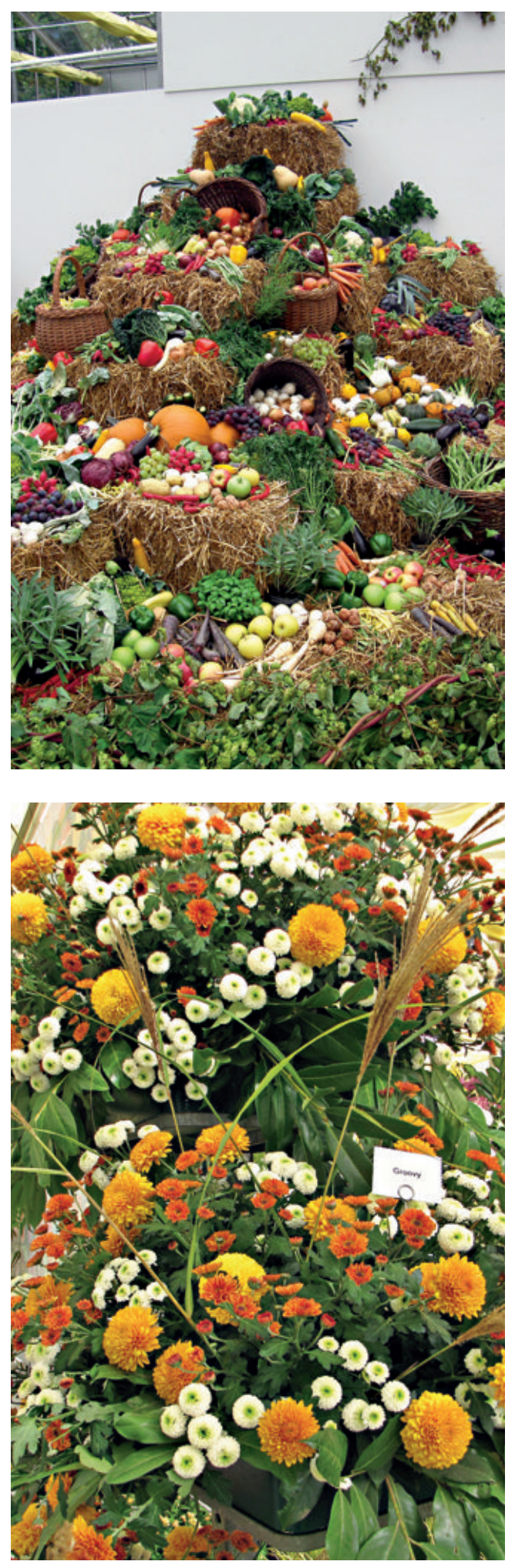

Kammersängerin JUNE CARD moderiert das feierliche Konzert mit Solisten, die Arien, Duette und Szenen aus den erfolgreichsten Werken PUCCINIs darbieten.

Stilvoller als mit Titi RoBINs hätte „Weltmusik im Palmengarten" wohl kaum zu Ende gehen können. Seine kulturenübergreifende Musik zwischen sanfter Melancholie und Überschwang trifft perfekt die Stimmung zum vorläufigen Abschied von der erfolgreichen Reihe bis zum Wiedersehen im Sommer 2010. Die Kulturregion stellt die Publikation „SIESMAYERs Gärten“ vor. Nicht nur im Palmengarten hat HeINRICH SieSMAYER seine Spuren hinterlassen. Er war im gesamten Rhein-Main-Gebiet tätig. Zahlreiche Parks tragen bis heute seine Handschrift. An sein vielseitiges Wirken erinnert jetzt der Band „SiesmaYers Gärten“ der Gartendenkmalpflegerin BARBARA VOGT.

JOHN HOLLENBECK und seine Band „ClAUdiA QUINTET“ kommen aus New York. Da gibt's den Central Park, aber keinen Palmengarten. Noch nie, so begrüßt HoLlENBECK die Zuhörer des letzten Jazz-Konzertes der Saison im Palmengarten, habe er an einem Ort ein Konzert gegeben, wo es dermaßen gut riecht. Der Zonta Club Frankfurt II Rhein-Main lädt zum BenefizKonzert in den Palmengarten. Ein „klassischmusikalisches Sommermärchen unter Palmen“, dessen Erlös an das FeM Mädchenhaus Frankfurt geht. Die ARD- und hr-Moderatorin Claudia SCHICK führt durch das Programm.

\section{September}

Bevor der graue Winter kommt, explodieren noch einmal die Farben, Dahlien und Astern blühen in Rot-, Gelb- und Lilatönen, Gräser und Blätter färben sich. Der Palmengarten präsentiert seine Erntedankausstellung in die aktuelle Ausstellung „Farbe in der Natur" integriert. Traditionell findet am Samstag der Erntedankschau das Herbstfest statt. Zum Abschluss steht ein Laternenumzug für Kinder auf dem Programm.

Abb. 6 (oben): In der Erntedankausstellung.

Abb. 7 (unten): Herbstliches Gesteck in der Chrysanthemen-Ausstellung. 
Der Magistrat trotzt der Wirtschaftskrise mit Investitionen, verschiebt aber auch einige Vorhaben. So wird auch der Bau des im Palmengarten geplante Blüten- und Schmetterlingshaus auf das Jahr 2012 geschoben. Der Zaun zur Zeppelinund Miquelallee soll zu einem vertikalen Garten werden. Der Tropenbotaniker und Künstler PATRICK BlanC, der Wände zum blühenden Leben erwecken kann, ist im Palmengarten zu Besuch. Eigentlich will er in einem Vortrag seine Arbeit und das Buch „Vertikale Gärten“ vorstellen. Seine vertikalen Gärten schmücken renommierte Hotels, Einkaufszentren, Flughafenwände, Parkhäuser, Brücken und Museen auf allen Kontinenten. Er lobt den Palmengarten als einen der bedeutendsten Botanischen Gärten Europas und entwickelt gleich erste Ideen für eine Mur végétale in Frankfurt. Eine grüne Wand soll es werden, die den Palmengarten vom Lärm und den Abgasen der Miquelallee abschirmt. Die Wand wäre mit über $1 \mathrm{~km}$ Länge die längste Pflanzenwand der Welt. Jetzt fehlt nur noch das Geld.

\section{Oktober}

Ein letztes Aufglühen der Natur kann in dieser Jahreszeit bewundert werden. Der Palmengarten integriert dieses Jahr auch die ChrysanthemenSchau in die Farbausstellung.

LEOPOld SONNEMANN gehört zu den bedeutendsten Köpfen Frankfurter Geschichte, dennoch kennen ihn nur wenige. Zum 100. Todestag widmet das Historische Museum dem Verleger, Politiker und Mäzen eine Ausstellung. Frankfurt hat SONNEMANN viel zu verdanken, so auch die Alte Oper, den Eisernen Steg und den Palmengarten. In der Ausstellung wird eine Originalaktie der Palmengarten-Gesellschaft gezeigt.

\section{November}

Der mit seinem Büro im Palmengarten ansässige Verein zum Schutz tropischer Lebensräume „Tropica Verde" feiert 20-jähriges Jubiläum und viele Erfolge. Heute zählt der Verein über 400 Mitglieder und kann auf erfolgreiche Projekte in Costa Rica blicken.

Abb. 8: Eine der vielen internationalen Krippen in der Weihnachtsausstellung.

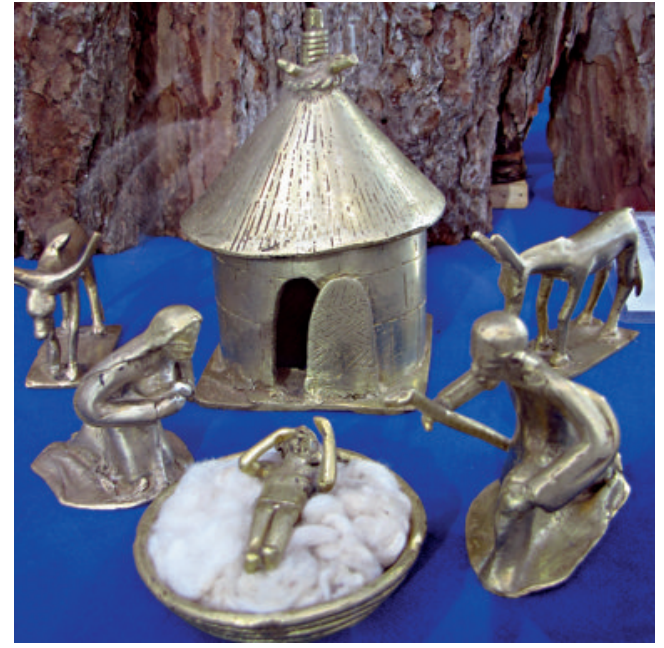

Im Rahmen der Veranstaltungs-Reihe des Dezernates für Umwelt und Gesundheit „Frankfurt is(s)t gut" bietet der Palmengarten eine Veranstaltung unter dem Motto „Alles Banane?“ an. Neben Führungen gibt es auch eine Diskussion zu Anbau, Handel und Genuss tropischer Früchte. Mit einem Aktionstag präsentiert sich das Pilotprojekt „Kinder im Garten“ der Öffentlichkeit. 150 Kinder zeigen ihren Eltern, was sie bereits gelernt haben. Die Arbeit trägt erste Früchte. Viele Eltern aus bildungsfernem Umfeld sind zum ersten Mal im Palmengarten und werden von der Begeisterung der Kinder für „ihren Palmengarten“ angesteckt. In den nächsten beiden Jahren sollen alle Frankfurter Kindergärten an dem Projekt teilnehmen können.

\section{Dezember}

In der Weihnachtsausstellung werden verschiedene Nadelbäume wie z.B. Blaufichte, WymouthKiefer, Douglasie und Colorado-Tanne gezeigt. Unter dem Motto Faszination Krippen zeigt der Palmengarten als erste Ausstellung in der neu renovierten Galerie Weihnachtskrippen aus aller Welt vom Krippen-Museum Nidda. Besonders interessant sind die Materialien, aus denen die Krippen hergestellt wurden. So gibt es Unikate aus Altmetall, Filz oder Ebenholz.

Dieser Jahresrückblick wurde anhand von Presseberichten über den Palmengarten zusammengestellt. 